\title{
Funktionale Differenzierung und gesellschaftliche Rationalität $\mathrm{Zu}$ Niklas Luhmanns Konzeption des Verhältnisses von Selbstreferenz und Koordination in modernen Gesellschaften
}

\section{Klaus Bendel}

Philipps-Universität Marburg, Institut für Soziologie, Am Grün 1, D-35037 Marburg

\begin{abstract}
Zusammenfassung: Niklas Luhmann hat unter dem Stichwort der Autopoiesis sozialer Systeme eine Theorie der modernen Gesellschaft entwickelt, die sich durch die Annahme einer operativ geschlossenen Selbstreproduktion funktional ausdifferenzierter Subsysteme auszeichnet. Vor diesem Hintergrund stelit sich die Frage, inwieweit die moderne Gesellschaft noch in der Lage ist, die Dynamik auseinanderstrebender Teilrationalitäten zu einem überlebensfähigen Gesamtzusammenhang zu integrieren und auf welche Ressourcen sie dabei zurückgreifen kann. Es soll versucht werden, dieses Problem entlang der Theorie Luhmanns zu rekonstruieren, um entgegen dessen weitreichender Interpretation operativer Geschlossenheit anhand der Unterscheidung von Code und Programm Bedingungen und Möglichkeiten intersystemischer Koordination aufzuzeigen und darüber hinaus auf der Grundlage einer spezifischen Verknüpfung von handlungs- und systemtheoretischer Perspektive Konsequenzen im Hinblick auf die Bewertung des Rationalitätspotentials moderner, funktional differenzierter Gesellschaften zu ziehen.
\end{abstract}

\section{Einleitung}

Die Soziologie beobachtet seit ihren Anfängen gesellschaftliche Entwicklung in aller Regel als einen Prozeß der zunehmenden Ausdifferenzierung von Teilbereichen, die sich von den Bindungen an ihr soziales Umfeld lösen, indem sie eine spezifische Selektivität der Handlungskoordination ausbilden.

Mit dieser Diagnose geht der Versuch einher, Momente in der Gesellschaft ausfindig zu machen, die in der Lage sind, die verschiedenen auseinanderstrebenden Teilrationalitäten der Subsysteme zu integrieren und auf diese Weise die Überlebensfähigkeit der Gesellschaft insgesamt sicherzustellen.

Durkheim (1988) etwa lokalisierte ein derartiges Potential in einer solidaritätsstiftenden Moral, Parsons (1968) in den Bindungswirkungen institutionalisierter Normen, Habermas (1981) in den Strukturen verständigungsorientierter Kommunikation.

Die Dynamik der Ausdifferenzierung gesellschaftlicher Teilbereiche in modernen, funktional differenzierten Gesellschaften scheint jedoch die gesellschaftsweite Wirkungskraft derartiger Integrationsmechanismen zunehmend zu erodieren und die These zu bestätigen, daß die Rationalisierungsprozesse der Moderne vorrangig das Moment der Spezialisierung und Verselbständigung zur Entfaltung bringen, während die kulturellen Sedimente gesellschaftlicher Solidarität hingegen lediglich als ein abhängiges Residuum dieser Art Fortschritt erscheinen, denen keinerlei Spielraum für autonome Normierungsprozesse bleibt.

Insbesondere Jürgen Habermas (1981) hat mit seiner Diagnose der 'Kolonialisierung der Lebenswelt' durch hochgradig selektiv operierende, mediengesteuerte Subsysteme der Gesellschaft diese These zur Diskussion gestellt. ${ }^{1}$ Der den Überlegungen von Habermas immanente Duktus kritischer Theorie zeichnet sich dabei dadurch aus, daß den seines Erachtens bedrohten originären Formen gesellschaftlicher Integration und Verständigung unter dem Stichwort einer 'Modernisierung der Moderne' wieder zu der ihnen angemessenen Geltung verholfen werden soll.

Hierzu sieht die neuere Systemtheorie weder Anlaß noch Chance.

Aus ihrer Perspektive erscheint die zunehmende Differenzierung der Gesellschaft nicht als ein einseitiger Rationalisierungsproze $\boldsymbol{B}$, dem eine pathologische Struktur anhaftet, insofern er fundamentale Grundlagen des sozialen Lebens untergräbt, sondern als ein Steigerungszusammenhang, der alle Bereiche der Gesellschaft erfaßt und in der Ausbildung operativ geschlossener Subsysteme kulmi-

1 Obgleich natürlich auch bereits klassische Denker wie Marx oder Weber, an die Habermas anknüpft, derartig einseitig strukturierte Rationalisierungsprozesse im Blick hatten. 
niert. Die Auflösung von Möglichkeiten gesellschaftsweiter Koordination signalisiert unter diesen Voraussetzungen keineswegs eine Krise der Moderne, sondern dokumentiert vielmehr die Entfaltung ihrer eigentümlichen Struktur, die sich von den Relikten einer vormodernen, hierarchisch gegliederten Ordnung befreit. ${ }^{2}$

In dem Maße, wie die funktionale Differenzierung sich zum primären Ordnungsprinzip der modernen Gesellschaft entwickelt, verbietet sich insofern zwangsläufig die Vorstellung, daß der soziale Gesamtzusammenhang von irgendeinem privilegierten Punkt aus, etwa durch Moral, Recht oder verständigungsorientierte Diskurse, in seiner Entwicklung bestimmt werden könnte. Konzepte einer hierarchischen Steuerung sind daher zu ersetzen durch die Annahme einer dezentralisierten, operativ geschlossenen Selbstreproduktion funktional ausdifferenzierter Teilsysteme.

Dieses vor allem von Niklas Luhmann unter dem Stichwort der Autopoiesis sozialer Systeme entwickelte Konzept der Gesellschaft (vgl. insbes. Luhmann 1984), das sich durch die Prinzipien der Heterarchie und Inkommensurabilität auszeichnet, wirft in seiner intersystemische Steuerungsund Koordinationsmöglichkeiten negierenden Radikalität die Frage auf, in welcher Form sich die Gesellschaft überhaupt noch als funktionsfähiger Gesamtzusammenhang, dessen Teilbereiche in einem konsistenten Verhältnis zueinander stehen, reproduzieren kann. Wie ist soziale Ordnung unter diesen Voraussetzungen möglich oder, um es in Luhmanns Worten zu formulieren, wie reproduziert sich die Gesellschaft als emergente, selbstsubstitutive Ordnung unter der Voraussetzung, $\mathrm{da} B$ ihre Teilbereiche und sie selbst als autopoietische Systeme füreinander Umwelten bilden, so daß sich keinerlei kausale Einflußmöglichkeiten eröffnen?

Das Problem von Struktur und Form der Beziehungen zwischen Systemen, die Sinn als gemeinsames Operationsschema nutzen, wurde bisher vorwiegend im Rahmen der Diskussion um das Verhältnis von Individuum und Gesellschaft bzw. psychischen und sozialen Systemen erörtert.

Luhmann hat in diesem Kontext gegenüber klassischen Konzeptionen der Intersubjektivität die These entwickelt, daß sich die Kommunikation keineswegs auf der Grundlage gemeinsam geteil-

2 Mit dieser Einschätzung unterscheidet sich die neuere Systemtheorie trotz vieler Parallelen von Theorien der sog. 'Postmoderne'. ter Wissensbestände, die eine Verständigung ermöglichen, vollzieht, sondern die Struktur doppelter Kontingenz vielmehr eine fundamentale Differenz impliziert, welche den Ausgangspunkt des emergenten Kommunikationsgeschehens markiert und dessen Eigenselektivität begründet. Nicht der intersubjektive Konsens sondern ein unüberwindbarer Dissens zeichnet die Beziehungen der Individuen im Rahmen der Kommunikation aus (vgl. exemplar. Luhmann 1984: $148 \mathrm{ff}$ ).

Hiergegen erhobene Einwände nehmen vor allem auf die sozialen Voraussetzungen der Ausbildung individueller Identität bezug und halten gegenüber Luhmanns Konzept der Interpenetration auf der Grundlage wechselseitiger Intransparenz an der Vorstellung eines Vermittlungs- bzw. Übertragungsmoments in der Kommunikation fest. ${ }^{3}$ Diese Kontroversen um das Problem der Kombination von operationaler Geschlossenheit und intersystemischer Koordination sollen im folgenden genutzt werden, um die Implikationen der funktionalen Differenzierung moderner Gesellschaften im Hinblick auf Möglichkeiten ihrer rationalen Selbstreproduktion näher zu beleuchten.

$\mathrm{Zu}$ diesem Zweck werde ich zunächst auf die besondere Wendung, die Luhmann dieser Fragestellung gegenüber klassischen soziologischen Handlungs- und Systemtheorien gibt, eingehen und versuchen, deren Aussagegehalt näher zu bestimmen (2.), um anschließend Formen intersystemischer Koordination auf der Grundlage der Selbstreferenz sozialer Systeme vorzustellen (3.) und hieraus abschließend Konsequenzen im Hinblick auf die Bewertung des Rationalitätspotentials moderner, funktional differenzierter Gesellschaften zu ziehen (4.).

Die These, die ich in diesem Rahmen versuchen werde zu erhärten, läßt sich dahingehend zusammenfassen, daß die funktionale Ausdifferenzierung operativ geschlossener gesellschaftlicher Teilsysteme Möglichkeiten gezielter sozialer Koordination und Beeinflussung grundsätzlich nicht ausschließt, sondern ihr entsprechende Formen intersystemischer Kopplung generiert, die modernen,

3 Im Anschluß an Mead und Wittgenstein vgl. etwa Giegel (1987), Miller (1987), Bender (1989). Die Diskussionen kreisen dabei nicht nur in diesem Rahmen um die Frage, in welcher Form der Umweltbezug und die Eigenselektivität sozialer Systeme zusammenwirken. Vgl. jüngst Martens (1991), Luhmann (1992) und Martens (1992), ergänzend auch die Vorschläge von Pokol (1990), Teubner (1987; 1989) sowie Willke $(1987 ; 1989)$. 
weithin dezentrierten Gesellschaften ein Potential an rationaler Selbstreproduktion erhalten.

\section{Die Autopoiesis sozialer Systeme}

\subsection{Zur Interpretation der operationalen Geschlossenheit von Sinn}

Die Spezifik der Überlegungen Luhmanns zu einem selbstreproduktiven Charakter sozialer Beziehungen findet ihren Ausgangspunkt in Defiziten, die seines Erachtens den klassischen Optionen der soziologischen Theoriebildung anhaften.

Sowohl handlungstheoretische ${ }^{4}$ als auch traditionell systemtheoretische ${ }^{5}$ Ansätze sind aus seiner Sicht nicht in der Lage, das Problem emergenter Effekte befriedigend zu handhaben, da sie sich nicht konsequent von kausalen, konstitutionstheoretischen Betrachtungsweisen lösen.

Während Handlungstheorien vor dem Problem stehen, Sozialität als eine Aggregation von Einzelhandlungen betrachten zu müssen, erscheinen die Sinnhorizonte handelnder Akteure im Kontext funktionalistischer Systemtheorien als ein vollständig durch die Strukturen des sozialen Zusammenhangs determiniertes Phänomen. Beide Theoriemodelle verfangen sich auf diese Weise entsprechend ihrer Bezugspunkte in den wechselseitigen Autonomiespielräumen von Ganzem und Teil, System und Element, Handlung und sozialer Ordnung oder Individuum und Gesellschaft.

Demgegenüber versucht Luhmann die These einer jeweils eigenständigen Selbstreproduktion psychischer und sozialer Systeme zu etablieren. Aus der Einsicht, daß sich die Operationen eines Sinnsy-

4 Hierzu zählt er alle Ansätze, die soziale Systeme als aus Handlungen aufgebaut betrachten und in dieser Weise den Mitteilungscharakter der Kommunikation bzw. den Bezug auf das Selbstverständnis der Akteure auszeichnen (vgl. Luhmann 1984: $191 \mathrm{ff}$ ). Entsprechend breit ist die Palette theoretischer Orientierungen, die unter diesen Begriff subsumiert werden. Sie reicht von der utilitaristischen Handlungstheorie der klassischen Nationalökonomie und Gesellschaftstheorie, etwa bei Thomas Hobbes und Adam Smith, über die von Weber, Mead und Schütz begründete Tradition einer sinnverstehenden Soziologie und die hieran anschließende Theorie kommunikativen Handelns von Habermas bis hin zu an die behavioristische Psychologie bzw. den klassischen 'homo oeconomicus' anknüpfenden Theorien der rationalen Wahl und spieltheoretischen Ansätzen.

5 Hier ist natürlich in erster Linie das Werk von Parsons zu nennen. stems nie vollständig aus den Einflußfaktoren der Umwelt erklären lassen, ist seines Erachtens der Schluß zu ziehen, daß diese ihre Identität aufgrund einer ihnen immanenten Eigenselektivität konstituieren und reproduzieren. Sie besitzen einen selbstreferentiellen bzw. autopoietischen Charakter, sofern sie trotz struktureller Umweltkopplungen ihre Einheit ebenso wie ihre Elemente durch relationierende Operationen ausschließlich selbst zustandebringen.

Kurz: Autopoietische Systeme sind das, was sie sind, durch sich selbst. Obwohl strukturell an ihre Umwelt gekoppelt, d.h. nie unabhängig von dieser existent, kann ihre Identität nicht durch außerhalb von ihnen liegende Instanzen sondern nur aufgrund ihrer Eigenselektivität hervorgebracht und reproduziert werden. ${ }^{6}$

Im Hinblick auf die Theorie sozialer Systeme entwickelt Luhmann hieran anschließend im wesentlichen zwei bedeutsame Konsequenzen:

a. Soziale Systeme reproduzieren sich ohne kausalen Bezug auf extern vorausgesetzte Elemente als Kommunikationssysteme.

Die Vorstellung, daß Akteure soziale Ordnung aufgrund eigener Überlegungen herstellen bzw. umgekehrt gesellschaftliche Normen die Gedanken der Individuen leiten, wird aufgegeben zugunsten einer wechselseitig unabhängigen Selbstreferenz. Sinn wird in psychischen und sozialen Systemen jeweils eigenständig in Form von Gedanken und Kommunikationsbeiträgen prozessiert. Kommunikation und Bewußtsein stehen somit nicht in einem Verhältnis von System und Element, sondern von System und Umwelt. Daraus folgt, daB die eine Seite in die Operationslogik der jeweils anderen weder eingreifen noch sie gar steuern kann.

b. In der modernen Gesellschaft gilt dies in gleichem Maße für das Verhältnis funktional ausdifferenzierter Teilsysteme.

Bereiche wie etwa Wirtschaft und Politik reproduzieren sich jeweils operational geschlossen mittels eigener symbolisch generalisierter Kommunikationsmedien (Geld und Macht) unabhängig von übergreifenden gesamtgesellschaftlichen Koordinationsinstanzen. In Form von Zahlungen oder

6 Vgl. exemplarisch Luhmann (1992), grundlegend Luhmann (1984), zum Begriff der Selbstreferenz insbesondere Luhmann (1984: $57 \mathrm{ff}$ ). Zur Theorie autopoietischer Systeme und Formen der Selbstorganisation im allgemeinen vgl. exemplarisch Maturana (1982), von Foerster (1985), Schmidt (1987), Krohn/ Küppers (1992). 
bindenden Entscheidungen konstituieren sich selbstreferentielle Kommunikationszusammenhänge als interne Umwelten der Gesellschaft.

Um Mißverständnissen bei der Erläuterung dieser beiden Annahmen vorzubeugen, muß zunächst jedoch die These einer operativen Geschlossenheit im Hinblick auf Sinnsysteme weitergehender spezifiziert werden, da man sie in zwei Versionen, einer schwachen und einer starken, interpretieren kann:

In ihrer schwachen Version bezieht sie sich ausschließlich auf die Form systemischer Operation und dürfte ebenso unstrittig wie trivial sein. $\mathrm{Da} B$ beispielsweise Bewußtseinssysteme zwar alles Mögliche, etwa die Natur, die Gesellschaft oder sich selbst, thematisieren, dies aber ausschließlich in der Form von Gedanken tun, kann wohl von niemandem ernsthaft in Zweifel gezogen werden. Im Bewußtsein findet nichts anderes Anschluß als ein neuer Gedanke an einen vorausgegangenen. Die 'unbiegsame Härte' (Luhmann) der systemischen Autopoiesis reduziert sich in diesem Fall auf die einfache, sicherlich nicht neue Erkenntnis, daß der 'Begriff des Hundes nicht bellt'.

Bei dieser Version läge es allerdings durchaus im Bereich des Möglichen, daß das, was im Bewußtsein in Form von Gedanken prozessiert wird, durch externe Ereignisse in der Umwelt, etwa durch die Beteiligung an der Kommunikation, zumindest mitbestimmt wird. Diese Möglichkeit ist hingegen in der 'starken', von Luhmann vertretenen Version grundsätzlich ausgeschlossen.

Bei dieser Variante sind kausale Einflüsse der Umwelt auf die Operationen des Systems im Sinne einer Übertragung oder Bewirkung von Wirkungen grundsätzlich nicht möglich, da autopoietische Systeme ihre Umwelt lediglich als unspezifische Reizung ('Rauschen') registrieren und ausschließlich selbst die Form ihres Umweltbezugs bestimmen. Sie legen durch ihre Eigenaktivität überhaupt erst fest, was sie als Umwelt wahrnehmen. ${ }^{7}$

Am Beispiel des Bewußtseins könnte man daher auch sagen, daß die operative Geschlossenheit sich nicht nur auf die Form der Operationen bezieht, sondern auch das, was im System AnschluB findet, einschließt. ${ }^{8}$ Kurz: der autopoietische Cha-

7 „Jedes System hat nur den Umweltkontakt, den es sich selbst ermöglicht..." (Luhmann 1984: 146).

8 „Anders gesagt: das Bewußtsein ist kein System, dessen Strukturen sich durch Input spezifizieren lassen... Vielmehr werden Umweltgegebenheiten nur als Irritation, als Störung, als Rauschen eingeführt, und dann intern nach Maßgabe eigener Strukturen spezifiziert" (Luhmann 1985a: 416f). rakter des Systems bestimmt nicht nur wie gedacht wird, sondern auch was gedacht wird. Nicht allein der Code, sondern auch die Programme sind rein selbstreferentiell produzierte Konstruktionen eines Systems. ${ }^{9}$

\subsection{Sonderformen intersystemischer Kopplung: Interpenetration und Interferenz}

Diese, die eigentliche Originalität des Autopoiesistheorems charakterisierende Interpretation operationaler Geschlossenheit wirft die Frage auf, wie es überhaupt zu Prozessen wechselseitiger Anpassung bzw. Co-Evolution, etwa zu Phänomenen der Inklusion und Sozialisation, zwischen Sinnsystemen kommen kann.

Daß die Kommunikation ohne die Beteiligung von mit Bewußtsein begabten Individuen nicht möglich ist, wird wohl von niemandem, auch nicht von Luhmann, bestritten. Ebensowenig dürfte die fundamentale Bindung des Bewußtseins an die Kommunikation zumindest im Bereich der Sozialwissenschaften seit den Arbeiten von Mead, Schütz. Wittgenstein u.a. in Frage gestellt werden.

9 Die Programme haben gleichsam nur eine dem Code untergeordnete Funktion, indem sie die selbstreferentielle Schließung des Systems entlang des Codes sichern und auf diesen Aspekt bezogen sind: „Die gesellschaftliche Ausdifferenzierung des Funktionssystems wird aber nicht über die Programmierung, sondern zunächst über die binäre Codierung erreicht... Das heißt: Der Selektionscode bewirkt die Ausdifferenzierung, und die Programme des Systems müssen dann von gesamtgesellschaftlichen Selbstverständlichkeiten abgekoppelt und an der Funktion, Codewerte zuzuordnen, ausgerichtet werden... Die Programme transformieren das bloße 'Rauschen' der Umwelt in einen für das System praktizierbaren Sinn“ (Luhmann 1987: 197 f). Es handelt sich demnach um eine Vorstellung, bei der Systeme nicht einfach nur mit einem eigenständigen Code auf Anforderungen der Umwelt reagieren. Die auf der Grundlage der Codes sich reproduzierende operative Geschlossenheit der Systeme generiert vielmehr selbst die Formen des Umweltbezugs. Das Kennzeichen der Wirtschaft etwa besteht unter diesen Voraussetzungen nicht in einer Befriedigung von Bedürfnissen, die aus der Umwelt an sie herangetragen werden. Vielmehr erscheinen Bedürfnisse als ein selbstkonditioniertes Moment des Wirtschaftssystems, mit dem dieses die Reproduktion seiner operativen $\mathrm{Ge}$ schlossenheit, d.h. seiner Orientierung an Zahlungen, sichert. Profitorientierung und zunehmende Relevanz der Befriedigung von Produktionsbedürfnissen symbolisieren diesen Aspekt (vgl. Luhmann 1988: 62f). 
Aus der Sicht Luhmanns ergibt sich der besondere wechselseitige Verweisungszusammenhang psychischer und sozialer Systeme aus der gemeinsamen Verwendung von Sinn als Differenzschema. Dadurch wird es möglich, da 3 Ereignisse, die einem konkreten Systemzusammenhang zugerechnet werden, zugleich Verwendung in einem anderen finden. Im Hinblick auf das Verhältnis psychischer und sozialer Systeme charakterisiert er diesen Sachverhalt mit dem Begriff der Interpenetration (vgl. Luhmann 1984: 286 ff; 1988a), ${ }^{10}$ im Hinblick auf das Verhältnis sozialer Systeme mit dem Begriff der Interferenz (vgl. Luhmann 1990: 298 f). ${ }^{11}$

Entscheidend ist in beiden Fällen, daß trotz der engen operativen Kopplung eine Seite die jeweils andere nicht determinieren kann (vgl. Luhmann 1990: 37-44).

Der in einer Kommunikation geäußerte Satz, „morgen soll es schönes Wetter geben“, kann als Anschlußbeitrag eine Äußerung des Gesprächspartners zum schlechten Wetter der vergangenen Tage hervorrufen, während er zugleich im Bewußtsein eines der Beteiligten die Idee eines möglichen Fahrradausflugs aufkommen lassen könnte. Diese Assoziation wird zwar durch die Kommunikation angestoßen, sie ergibt sich aber allein aufgrund der besonderen internen Verweisungen des entsprechenden psychischen Systems und konnte aufgrund der von außen nicht einsehbaren Binnenkomplexität vom Gesprächspartner allenfalls erwartet, nicht jedoch festgelegt werden.

Die zeitgleiche Dynamik, die ein Ereignis in beide Richtungen unter jeweils systemspezifischen Kriterien auslöst, schließt es trotz permanenter wechselseitiger Einflüsse aus, daß eine Seite die andere in ihrer Selektivität Punkt für Punkt führt.

Während man einen Gedanken ausspricht schießt bereits das eigene Bewußtsein weit über das hinaus, was eine Anschlußkommunikation des Gesprächspartners noch beeinflussen könnte. So regt dessen Antwort zwar wiederum neue eigene Gedanken an, den Assoziationsreichtum des Bewußtseins jedoch kann die Kommunikation zu keinem Zeitpunkt unter ihre Kontrolle bringen.

Gleiches gilt im umgekehrten Fall. Der Versuch eines Bewußtseinssystems, die Kommunikation in

10 Eine Referenz an Parsons, dessen diesbezügliche Vorstellung einer wechselseitigen Durchdringung und Überlappung von Systemen jedoch kaum mehr etwas mit der Interpretation Luhmanns gemein hat.

11 Teubner (1989: $102 \mathrm{ff}$ ) hat diesen Begriff zu von Luhmanns Auffassung abweichenden Schlußfolgerungen genutzt. ihrem Verlauf zu kontrollieren, erscheint aufgrund deren Schnellebigkeit als ein aussichtsloses Unterfangen.

Bei einem Streit etwa überlegen die Beteiligten in der Regel erst nachträglich, was sie in der Hitze eines Wortgefechts eigentlich gesagt haben. Die Antworten des anderen erscheinen viel eher als die Stichwortgeber der eigenen Kommunikationsbeiträge als die vorausgegangenen eigenen Gedanken. Man hat in erster Linie die Aussagen des Gesprächspartners und weniger den diffusen Gedankenreichtum des eigenen Bewußtseins vor Augen. Der Sprung von einer Äußerung zur nächsten liegt näher und verläuft reibungsloser als der mühsame (durchaus nicht blockierte) Weg der Übersetzung eines Gedankens in die Kommunikation.

So bringt man zwar permanent Gedanken in die Kommunikation ein und die Kommunikation reagiert hierauf, sie entwickelt jedoch grundsätzlich eine Eigendynamik, die das Bewußtsein bei seinen Kontrollversuchen hoffnungslos 'hinterherlaufen' läßt. ${ }^{12}$ Der Grund für den Anschluß von Kommunikationen an Kommunikationen kann daher nur in der Kommunikation selbst liegen und nicht in den sicherlich unabdingbaren Beiträgen, die das Bewußtsein für das Zustandekommen der Kommunikation erbringt.

Obwohl Kommunikation und Bewußtsein ununterbrochen die Leistungen des jeweils anderen Systemtyps zum Aufbau eigener Ordnung nutzen ${ }^{13}$ und sich in dieser Form durch einen Rückgriff auf systemextern bereits vorstrukturierte Komplexität von Eigenaktivitäten entlasten, unterscheidet sich die Struktur des wechselseitigen Umgangs mit diesen Leistungen grundsätzlich nicht von der Resonanz anderer Umweltirritationen.

Ebenso wie neuronale Reize erst durch das Gehirn selbst eine Spezifizierung als 'Sehen', 'Fühlen' oder 'Riechen' erfahren, läßt sich auch das Bewußtsein durch die Kommunikation lediglich irritieren, d.h. es muß den als 'Geräusche' registrierten Kommunikationsbeiträgen selbst einen Sinn zuweisen, um sie als solche zu identifizieren und eigene gedankliche Anschlußmöglichkeiten zu erzeugen. Dieser Sinn wird daher nicht durch die

12 Es ist etwa so wie beim Wettlauf zwischen Hase und Igel: Obwohl der eine fest davon überzeugt ist, dem anderen vorauszueilen, ist der andere immer schon am Ziel angelangt.

13 Die Kommunikation stützt sich auf die Wahrnehmungsfähigkeit des Bewußtseins und das Bewußtsein auf die Ordnungsmöglichkeiten der Sprache (vgl. Luhmann 1990: $565 \mathrm{f}$ ). 
Kommunikation in Form einer Übertragung mitgegeben, sondern erscheint als eine aus rein internen Relationierungen erzeugte systemspezifische Nachfolgeaktualiät: als Gedanke, der unabhängig von der Kommunikation an Gedanken anschließt.

In gleicher Weise produziert umgekehrt die Kommunikation ihre Anschlußbeiträge selbst. Sie stellen keine 'Einspeisungen' psychischer Systeme dar. Ihr emergenter Charakter ergibt sich daraus, daß eine von einer gedanklichen Inspiration getragene Äußerung lediglich auf Unterstellungen und Erwartungen im Hinblick auf die Kommunikationspartner beruht, so daß nicht sichergestellt werden kann, ob bzw. wie diese in der Kommunikation verstanden werden wird. Die Äußerung gewinnt ihre kommunikative Eigenschaft nicht bereits durch sich selbst bzw. durch den ihr zugrundeliegenden Gedanken, sondern erst dann, wenn eine Anschlußkommunikation ihr den Status einer informativen Mitteilung zuweist und sie dadurch als ein kommunikatives Ereignis behandelt. Diese Zuschreibung (das Verstehen) und nicht die mit einer Äußerung ursächlich verbundenen Absichten schafft den sozialen Bezug. ${ }^{14}$ Auch die aus dem Verstehen resultierende Anschlußkommunikation wird wiederum von den gleichen Unterstellungen und Projektionen getragen wie die vorausgegangene Kommunikationsofferte, so daß die hier zugrundeliegenden Leistungen des Bewußtseins gleichfalls keine die Kommunikation führende Qualität implizieren.

Entscheidend für die Eigenselektivität ist somit nicht allein, daß Ereignisse unter jeweils systemspezifische Bedingungen der Anschlußfähigkeit gestellt werden, sondern da $B$ ihr Charakter als ein für die systemische Selbstreproduktion relevanter Gesichtspunkt ausschließlich aus der bezugnehmenden Selektivität einer Nachfolgeaktualität resultiert. Es existiert insofern kein gemeinsames Ereignis, das die Beobachtungen von Kommunikation und Bewußtsein im Sinne eines für beide identischen Irritationsfaktors dirigiert. Vielmehr handelt es sich lediglich um eine zeitgleiche Konstitution von Ereigniszügen (Formen) in einem gemeinsamen Medium (Sinn) unter der Bedingung erhöhter wechselseitiger Resonanz. Auch die Sprache als Interpenetrationsmedium ermöglicht keinerlei intersystemischen Transport von Infor-

14 „Kommunikation kann als Mitteilung auf Verstehen hin angelegt sein und trotzdem scheitern. Trotzdem organisiert sie sich, wenn man so sagen darf, vom Verstehen aus..." (Luhmann 1986a: 95, vgl. auch Luhmann 1984: 212). mationen, sondern bietet Sinnsystemen sozusagen nur die Möglichkeit, die Ordnungsleistungen eines anderen Sinnsystems zu nutzen, um ihnen einen eigenen, hiervon unabhängigen Sinn zuzuweisen (vgl. Luhmann 1990: $37 \mathrm{ff}$, 565f; 1987a: $174 \mathrm{f}$; 1987c: 342f, 1984: 292 f; Fuchs 1992: 182f). ${ }^{15}$

Die Co-Evolution von Kommunikation und Bewußtsein kommt somit dadurch zustande, daß beide sich permanent Leistungen zur Verfügung stellen, ohne damit jeweils das andere System in seiner Selektivität kontrollieren zu können. Mit dem Begriff der Co-Evolution werden insofern zwar intersystemische Einflüsse und Anpassungsprozesse zugestanden, er bezeichnet jedoch gerade deren 'Blindheit' in Abgrenzung zu Formen gezielter Koordination und Steuerung. ${ }^{16}$

Diese Struktur der Beziehung zwischen psychischen und sozialen Systemen im Rahmen des Interpenetrationskonzepts läßt sich wiederum auf das Verhältnis gesellschaftlicher Teilsysteme übertragen:

Die Politik etwa benutzt Geld und Recht zur eigenen Selbstreproduktion, aber sie tut dies ausschließlich im Rahmen eines hiervon unabhängigen Machtkreislaufs nach eigenständigen Regeln unter Gesichtspunkten des Machtgewinns und der Machterhaltung. Sie operiert nicht nach den Kriterien wirtschaftlicher Rentabilität, obwohl sie über ihre finanziellen Etats an die ökonomischen Reproduktionsprozesse gebunden bleibt. Die Politik kann daher die finanziellen Aspekte ihrer Entscheidungen nicht ignorieren, sie ordnet diesen Aspekt jedoch der ihr eigentümlichen Operationslogik unter. Gleiches gilt auch für die Verwendung von Geld zur Produktion neuer Erkenntnisse in der Wissenschaft.

Aufgrund dieser Form der selbstreferentiellen Orientierung ist es diesen Systemen zugleich unmöglich, Ereignisse in anderen Bereichen im Sinne einer gesellschaftlichen Steuerung vorwegzubestimmen.

15 Siehe zu diesem Punkt auch die Kontroverse zwischen Martens (1991; 1992) und Luhmann (1992).

16 Die Bindungen zwischen Kommunikation und Bewußtsein werden durch Luhmanns Konzept der Interpenetration daher keineswegs einfach gelockert. Ihre wechselseitigen Verweisungen sind aufgrund der permanenten Zeitgleichheit vielmehr derart weitreichend und komplex, daß sie mit dem Kausalmodell von Vorher und Nachher bzw. Ursache und Wirkung nicht hinreichend beschrieben werden können (vgl. Luhmann 1990: 39; Baecker 1992: 227 f). 
Wirtschaftspolitische Maßnahmen resultieren aus den eigenständigen Bedingungen und Relevanzen des politischen Systems, während ihre ökonomischen Effekte sich hiervon unabhängig im Rahmen selbstreferentiell verknüpfter Zahlungsprozesse bilden (vgl. Luhmann 1988: 346f), neue Erkenntnisse finden in der Wirtschaft nicht deshalb Verwendung, weil sie neu bzw. wahr sind, sondern nur sofern sie eine Steigerung der Profitabilität von Unternehmungen erwarten lassen.

Die Politik kann Forschungsprogramme auflegen und auch die Wirtschaft kann hierfür finanzielle Mittel zur Verfügung stellen, aber sie können beide dadurch nicht unmittelbar neue Erkenntnisse produzieren. Dies geschieht ausschließlich im Rahmen eines eigenständigen Kommunikationszusammenhangs, der sich am Code wahr/unwahr orientiert. Politik und Wirtschaft sind zwar aufgrund ihrer operativen Kopplung an die Wissenschaft in der Lage, diese zu Aktivitäten zu veranlassen, aber sie können die entsprechenden Resultate nicht festlegen, etwa im Sinne: „eine Mark für eine Wahrheit" (Luhmann 1990: 638) ${ }^{17}$ Obgleich die Wissenschaft auf wirtschaftliche und politische Unterstützung angewiesen ist. lassen sich Wahrheiten weder kaufen noch erzwingen. Zahlungen und bindende Entscheidungen können die Eigenselektivität der wissenschaftlichen Kommunikation nicht ersetzen, allenfalls aufheben.

Die selbstreferentielle Geschlossenheit eines Systems kann aus der Sicht Luhmanns daher ausschließlich wirksam oder nicht wirksam sein. Es gibt keine dazwischenliegenden Möglichkeiten der Kombination von Geld oder Macht mit Wahrheit, so daß die Vorstellung einer graduellen Selbstreferenz von Systemen grundsätzlich ausgeschlossen ist. $^{18}$

Als Konsequenz ergibt sich daraus, daß psychische und soziale Systeme aufgrund ihres autopoietischen Charakters weder von außen gesteuert werden können noch diese selbst dazu in der Lage sind, über ihre Systemgrenzen hinweg in ihre Um-

17 Vgl. auch Luhmann (1991: $90 \mathrm{f} ; 1987 \mathrm{c}: 342 \mathrm{f}$ ), am Beispiel der strukturellen Kopplung von politischem System und Rechtssystem Luhmann (1990a: $193 \mathrm{ff}$ ).

18 „Der Begriff der Autopoiesis... hat eine gänzlich unbiegsame Härte. Ein System setzt seine Reproduktion fort oder es setzt sie nicht fort. Es gibt keine dazwischenliegenden Möglichkeiten. Eine Frau ist schwanger oder sie ist nicht schwanger, sie kann nicht ein bißchen schwanger sein“" (Luhmann 1985: 2). $\mathrm{Vgl}$. demgegenüber Gradualisierungsversuche etwa von Teubner $(1987 ; 1989)$. welt einzugreifen. Steuerung ist immer Selbststeuerung, Konditionierung immer Selbstkonditionierung.

\section{Intersystemische Koordination}

\subsection{Die Kombination von Offenheit und Geschlossenheit und die Unterscheidung von Code und Programm}

Einwände, die sich gegen dieses Konzept der Interpenetration bzw. Interferenz richten, machen hingegen geltend, da $\beta$ aufgrund der Verwendung des gleichen Differenzschemas (Sinn) Übertragungsprozesse zwischen sozialen bzw. psychischen und sozialen Systemen durchaus möglich sind.

Im Unterschied zu anderen Umweltbeziehungen können Sinnsysteme wechselseitig ihre Operationen in der Weise in ihre Selbstreproduktion einbauen, daß sie von interpretativen Eigenleistungen entlastet werden. Neuronale Reize bedürfen im Gehirn zunächst einer Zuschreibung, ob es sich um 'Sehen', 'Hören', 'Fühlen' oder 'Riechen' handelt. Hingegen können Gedanken an den in der Kommunikation artikulierten Sinn unmittelbar anschließen.

In einem nochmals gesteigerten Maße gilt dies für die Kommunikation gesellschaftlicher Teilsysteme: Jede spezialisierte Kommunikation in einem beliebigen Teilsystem, seien es Wahrheitsdiskurse, Zahlungen oder bindende Entscheidungen ,ist immer zugleich - buchstäblich uno actu - allgemeingesellschaftliche Kommunikation." (Teubner 1989: 107).

Daraus läßt sich der Schluß ziehen, daß Sinnsystemen aufgrund ihrer engen operativen Kopplung ein wechselseitiges Einflußpotential zugeschrieben werden kann, das über den Charakter rein äuBerer Irritation hinausreicht. Indem die Leistungen eines Systems in die Selbstreproduktion eines anderen unmittelbar einfließen, beeinflussen Sinnsysteme wechselseitig ihre interne Selektivität.

Die trotz einer engen operativen Bindung nach wie vor fortbestehende Eigenselektivität des jeweiligen Systems veranlaßt Luhmann jedoch, auch in diesen Fällen streng am Paradigma der Autopoiesis festzuhalten. Sinnsysteme erzeugen aus seiner Sicht unter Umständen ein höheres $\mathrm{Ma}$ an wechselseitiger Resonanz; aber kein Bereich greift in die Reproduktion eines anderen ein. Die Identität eines Zusammenhangs kann sich nur durch sei- 
ne Selbstreferenz und nicht durch externe Einflüsse reproduzieren (vgl. Luhmann 1990: 37). ${ }^{19}$

Neue Erkenntnisse der Wissenschaft etwa können Wirtschaftsunternehmen nicht dazu anregen, Rentabilitätskalküle aufzugeben und ihre Investitionsentscheidungen entlang des Codes wahr/unwahr zu orientieren. In welchen Fällen neue Technologien in die Produktion Eingang finden, entscheidet sich ausschließlich nach ökonomischen Kriterien. Die Wirtschaft 'benutzt' die Wissenschaft daher nur nach Maßgabe des eigenen Codes (Zahlen/Nichtzahlen). Gerade dadurch unterscheidet sie sich von ihrer Umwelt als eine eigenständige, autopoietische Ordnung.

Demzufolge können trotz der interferentiellen Kopplung sozialer Systeme die Elemente des einen nicht unmittelbar in die Reproduktion des anderen eingebaut werden. Es bedarf auch in diesen Fällen einer Eigenleistung des jeweiligen Reproduktionszusammenhangs. Was als Element eines Systems fungiert, wird nur durch dieses selbst festgelegt.

Diese These erscheint allerdings nur im Hinblick auf die als 'schwache Version' gekennzeichnete Interpretation des Autopoiesistheorems haltbar. ${ }^{20}$ Die 'starke Version' hingegen erfährt eine entscheidende Relativierung.

Trotz eines eigenständigen Codes können externe Ereignisse, wie Luhmann selbst anerkennt (vgl. Luhmann 1987b: 197f; 1988: 346f; 1990: 639; 1991a: 143 f), ${ }^{21}$ die Programme eines Systems offenbar in unterschiedlichem Ausmaß beeinflussen.

So kann beispielsweise die Politik Mittel zur Erforschung der Solar-, Wind- oder Kernenergie vergeben. Dies geschieht natürlich immer nur auf der Grundlage spezifischer politischer Eigeninteressen, d.h. unter dem Gesichtspunkt der Reproduktion von Macht, während zugleich damit die am Code wahr/unwahr orientierte Kommunikation des Wissenschaftssystems nicht aufgehoben werden kann. Gleichwohl wird dadurch jedoch dessen programmatische Orientierung beeinflußt. Die Politik legt mit ihren Entscheidungen fest, in welche Richtungen Wahrheiten gesucht werden.

19 Siehe bereits Abschnitt 2.2.

20 Unabhängig von Umweltereignissen bleibt der Code operativ geschlossen: Zahlungen knüpfen immer nur an Zahlungen an und Gedanken immer nur an Gedanken.

$21 \mathrm{Vgl}$. entsprechend auch im Hinblick auf das Verhältnis psychischer und sozialer Systeme Luhmann (1990: 27, 39).
Auch die Wirtschaft operiert selbständig nach den ihr eigenen Rentabilitätskriterien unabhängig von politischen Machtgesichtspunkten, aber rechtliche Nutzungsbeschränkungen und Auflagen bzw. steuerliche Vergünstigungen und Subventionen können Zahlungsentscheidungen in die eine oder andere Richtung beeinflussen. Ähnlich tangieren neue wissenschaftliche Erkenntnisse die programmatische Orientierung des Wirtschaftssystems und ökonomische Entwicklungen berühren wiederum die programmatische Orientierung der Politik. Analog wirkt die Kommunikation über Erziehungs- und Sozialisationsprozesse auf die Gedankengänge der Individuen, während diese auch umgekehrt dazu in der Lage sind, den Verlauf der Kommunikation mitzubestimmen.

Die ausschließliche Orientierung auf die Eigenselektivität und Selbstkonditionierung eines Systems verstellt an diesem Punkt eher den Blick auf den Umfang und den Charakter fremdreferentieller Einflüsse.

\subsection{Reflexive Kontextsteuerung}

Gesteht man aus diesem Grund externen Ereignissen ein Einflußpotential auf die Selbstreproduktion autopoietischer Ordnungen trotz deren operativer Geschlossenheit zu, stellt sich daran anschlieBend allerdings das Problem, wie es einem System gelingen kann, die selbstreproduktiven Leistungen eines anderen Systems so anzuregen, daß die von ihm erwarteten Wirkungen auch eintreten, ohne daß dies auf eine unmittelbare Fremdsteuerung nach dem Muster der Beeinflussung von Trivialmaschinen hinausläuft.

Wie sind intendierte intersystemische Einflüsse trotz der Eigenselektivität jeweils spezifischer binärer Codes möglich?

Dieses Frage verweist auf das Modell einer 'indirekten Kontextsteuerung' aufgrund der reflexiven Struktur von Sinn (vgl. Willke 1987; 1989: $111 \mathrm{ff}$; Eichmann 1989).

Im Unterschied etwa zu organischen bzw. neurophysiologischen Ordnungen sind psychische und soziale Systeme nicht nur in der Lage, ihre Elemente selbst zu bilden und in Beziehung zu setzen, vielmehr können sie diesen Prozeß basaler Selbstreferenz nochmals beobachten und als Baustein der Selbstrelationierung nutzen. In sozialen Systemen kann über Kommunikationen kommuniziert werden, in psychischen Systemen über Gedanken nachgedacht werden. Zugleich können sie sich selbst ebenso wie andere Systeme als relationie- 
rende Einheit beobachten und darüber hinaus auch die Rückwirkungen ihrer Umwelteinwirkungen auf sich selbst in Rechnung stellen. ${ }^{22}$ Sinnsysteme können also kurz gesagt sich selbst ebenso wie andere Systeme als Einheit in Differenz zu ihrer Umwelt beobachten.

Diese Fähigkeit eröffnet ihnen im Hinblick auf intersystemische Einflußbereiche die Möglichkeit, sich als Umwelt anderer Systeme zu betrachten und die eigenen Operationen unter dem Gesichtspunkt ihrer externen Wirkungen zu gestalten. Ohne ihre jeweiligen Codes zu überspringen und direkte Kontaktbereiche herzustellen sind psychische und soziale Systeme in der Lage, im Zuge der eigenen Selbstreproduktion in anderen Systemen gezielt Resonanzen zu erzeugen.

Die Politik etwa kann im Interesse der eigenen Machterhaltung wissenschaftliche Forschungsprogramme auflegen, Schadstoffgrenzwerte für die Produktion in der Wirtschaft erlassen oder Arbeitsbeschaffungsmaßnahmen beschließen. Sie operiert in diesem Fall insofern rational, als sie ihre Wirkung auf andere Funktionsbereiche anhand der Rückwirkungen auf den Machtkreislauf in Rechnung stellt.

Luhmanns Skepsis gegenüber den Möglichkeiten einer indirekten Kontextsteuerung begründet sich aus der operativen Geschlossenheit und der hiermit verbundenen Binnenkomplexität der gesellschaftlichen Teilsysteme.

Da die Entwicklungsdynamik eines Systems nie vollständig kalkulierbar ist und immer dann, wenn sich um Planung und Kontrolle bemüht wird, zeitgleich bereits milliardenfach etwas neues passiert, treten die Effekte eines noch so anspruchsvollen Steuerungshandelns zwangsläufig ungesteuert auf (vgl. Luhmann 1988: 324 ff; 1989). Reflexive Mechanismen tangieren insofern nicht den Charakter der Intransparenz intersystemischer Beziehungen, sondern stellen lediglich eine gesteigerte Form der Selbstreferenz dar, ohne die Grenze zwischen System und Umwelt zu relativieren. Sie schaffen keinerlei intersystemische Kontaktbereiche oder Austauschbeziehungen.

Forschungsförderung, Grenzwertfestlegungen oder Arbeitsbeschaffungsmaßnahmen markieren

22 Luhmann unterscheidet diese verschiedenen Stufen des Selbstbezugs mit den Begriffen der Reflexivität, der Reflexion und der Rationalität. Die Beobachtung der Selbstreferenz eines anderen Systems bezeichnet er als Verstehen (vgl. Luhmann 1984: $593 \mathrm{ff}$; 1986).
Selbstkonditionierungen des politischen Systems und keine Eingriffe der Politik in andere gesellschaftliche Teilbereiche. Derartige Maßnahmen werden ausschließlich im Rahmen machtförmiger Kommunikationsbeziehungen hervorgebracht, ohne daß ihnen eine darüber hinausgehende substantielle Bindung an neue Erkenntnisse, unbelastete Natur oder wirtschaftlichen Wohlstand anhaftet, während die Reaktionen der jeweiligen Funktionsbereiche auf die politischen Programme sich wiederum ausschließlich durch deren spezifischen Kommunikationscode bestimmen.

Nun ist sicherlich nicht zu bestreiten, daß politische Maßnahmen aufgrund massiver Eigeninteressen politischer Akteure an Machtgewinn bzw. Machterhaltung ergriffen werden und sich mit ihnen allenfalls sekundär die Sorge um andere $\mathrm{Be}$ reiche der Gesellschaft verbindet. Da jedoch, wie bereits deutlich wurde, durchaus die Möglichkeit intersystemischer Beeinflussung auf der Ebene der Systemprogramme unabhängig von der operativen Geschlossenheit spezifischer Kommunikationscodes besteht, eröffnen reflexive Mechanismen die Chance, fremdreferentielle Sachverhalte durch Beobachtung zur Selbstreproduktion gezielt zu nutzen.

Die Politik etwa kann aus eigenem Interesse versuchen, die Wirtschaft anzukurbeln oder den Umweltschutz zu verbessern. Die Binnenkomplexität anderer Systeme schließt dabei keineswegs aus, daß beabsichtigte Effekte mit hoher Wahrscheinlichkeit auch eintreten.

Wie sollte man ansonsten die Tatsache interpretieren, daß die Wirtschaft bleifreies Benzin anbietet, wenn die Politik das so vorschreibt, ${ }^{23}$ die Kenntnisse über HIV-Infektionen sich erweitern, wenn entsprechende Forschungsprogramme beschlossen werden oder die Arbeitslosenzahlen sinken, wenn eine aktive Arbeitsmarktpolitik betrieben wird. ${ }^{24}$ Der Aufbau von Erfahrungswerten bei der Beobachtung fremdreferentieller Sachverhalte eröffnet Chancen komplexitätsreduzierender Gegenstrategien, ohne die Differenz zwischen den Systemen aufgrund ihrer jeweiligen Eigenselektivität aufhe-

23 Ein Beispiel von Scharpf (1989: 12).

24 Wie bereits erwähnt bestreitet Luhmann derartige Effekte durchaus nicht (vgl. illustrativ am Beispiel eines Impfgesetzes Luhmann 1991a: 143 f). Im Unterschied zur hier vertretenen Auffassung verbindet sich seines Erachtens hiermit jedoch keine Relativierung des autopoietischen Charakters der Reproduktion sozialer Systeme. 
ben zu können. ${ }^{25}$ Zusätzliche Kenntnisse und Erwartungssicherheiten offenbaren zwar immer zugleich auch eine erweiterte Dimension der Unkenntnis und damit einhergehend neue Überraschungen und mögliche Enttäuschungen. ${ }^{26}$ Dies schließt jedoch temporäre Erfolge bei der Bemühung um eine intersystemische Koordination und Steuerung nicht grundsätzlich aus. Im Gegenteil: aus der Sicht der beteiligten Systeme handelt es sich dabei um ein unabdingbares Erfordernis ihrer Selbstreproduktion. Sie konstituieren und stabilisieren ihre Identität nur durch den Aufbau und die Bestätigung von Erwartungen im Rahmen einer Beteiligung an intersystemischen Kommunikationsprozessen.

Die Beschränkung der Perspektive auf systemische Selbststeuerungsmechanismen bleibt insofern zwar im Hinblick auf fehlende Kontaktbereiche und Determinationsbeziehungen zutreffend, sie ist jedoch weder in der Lage, die Gründe für das unaufhörliche Auftreten von intersystemischen Koordinationsbemühungen noch die Bedingungen ihrer möglichen Erfolge hinreichend zu berücksichtigen. ${ }^{27}$

\section{Gesellschaftliche Rationalität}

\subsection{Zum analytischen Potential der Unterscheidung einer handlungs- und systemtheoretischen Perspektive}

Die an diesem Punkt notwendig erscheinende Ergänzung des Konzepts kann sich jedoch nicht allein auf die Unterscheidung von handlungsfähigen und handlungsprägenden Sozialsystemen gründen (vgl. Schimank 1985, 1988). ${ }^{28}$

25 ,Verstehen (im Sinne der Rekonstruktion der Selbstbeschreibung des intervenierten Systems) macht die Wirkungen der Intervention zwar immer noch nicht beherrschbar, aber doch kalkulierbar - der Übergang von Sicherheit auf Risiko verringert das Risiko der Sicherheit: 'no risk is the highest risk of all'“ (Willke 1987: 351).

26 Vgl. hier im Hinblick auf die Stellung der Individuen zur Kommunikation Luhmann (1990: $22 \mathrm{f}$ ).

27 Es bleibt gleichsam ungeklärt, aus weichen Gründen Sinnsysteme sich wechselseitig permanent wider besseres Wissen als 'Trivialmaschinen' behandeln. Vgl. hierzu im Hinblick auf das Erziehungssystem Luhmann (1987: $179 \mathrm{f}$ ).

28 Hierzu analog sind Versuche zu sehen, individuellen oder kollektiven Akteuren aufgrund einer ihnen eigenen sog. 'multilingualen Kommunikationskompetenz' besondere Integrations- und Koordinations-
Sicherlich muß der Gesellschaft und ihren funktional ausdifferenzierten Teilsystemen gegenüber Individuen, Gruppen oder Organisationen der Status einer Handlungseinheit als Bezugspunkt der Kommunikation abgesprochen werden, sofern es ihnen nicht gelingt, sich konkurrenzfrei als adressable Identität zu repräsentieren (vgl. Luhmann 1991: 172f; 1986: 252f; Fuchs 1992: $225 \mathrm{f}$ ). Sie erscheinen in ihrer Struktur polykontextural. d. h. von verschiedenen Standpunkten aus jeweils unterschiedlich beobachtbar. Die Kommunikation behandelt diesen Typus sozialer Systeme daher nicht als Akteure, denen bestimmte Absichten, Interessen und Strategien der Zielerreichung unterstellt werden könnten.

Obwohl sie insofern nicht handlungsfähig sind und von daher auch zu intentionalen Steuerungsleistungen nicht in der Lage scheinen, üben sie gleichwohl einen dirigierenden Einfluß auf die von ihnen konstituierten elementaren Handlungseinheiten aus. Sozialen Systemen kommt unabhängig von ihrer Handlungsfähigkeit im oben beschriebenen Sinne eine handlungsmotivierende Qualität $\mathrm{zu}$, durch die es ihnen gelingt, sich als eine emergente Ordnung zu reproduzieren. Entscheidend für die Analyse intersystemischer Koordination ist daher nicht das Attribut der Handlungsfähigkeit ${ }^{29}$ sondern die Eigenschaft sozialer Systeme, im Rahmen ihrer Selbstreproduktion emergente Formen rationaler Kommunikation hervorzubringen. Im Vordergrund steht demnach nicht die Frage, wer Steuerungshandeln ausführt - hierzu sind per De-

funktionen zuzuweisen (vgl. etwa Mayntz 1987; Scharpf 1989). Die folgenden Einwände richten sich im Prinzip gegen alle Ansätze, die die Beziehung zwischen System und Handlung im Sinne eines dualistischen Restriktionsverhältnisses konzipieren (vgl. hierzu Heidenescher 1992). Wie ich im weiteren erläutern möchte, halte ich es für aussichtsreicher, diese Eigenschaften der Sprache als alltagsweltlichem Kommunikationsmedium im Rahmen eines emergenten, gesamtgesellschaftlichen Sozialzusammenhangs zuzuschreiben und sie nicht mit dem Attribut der Handlungsfähigkeit zu verknüpfen.

29 Die Differenzen hinsichtlich dieses Gesichtspunktes erscheinen ohnehin graduell und vom Stand sozialer Differenzierungsprozesse abhängig. Die Zurechnung von Handlungen, etwa auf Personen, fungiert lediglich als ein Mechanismus der Reduktion von Komplexität um Anschlußmöglichkeiten zu gewinnen und ist durch keinerlei substantielle Eigenschaften der hierdurch bezeichneten Handlungseinheit vorgegeben. Sie besitzt einen ausschließlich durch den Kommunikationszusammenhang konstruierten Charakter (vgl. Luhmann 1984: 229). 
finition nur individuelle oder kollektive Akteure in der Lage -, sondern wie bzw. wodurch spezifische Operationsmodi von Sinnsystemen erzeugt werden, welche die Rückwirkungen von Umweltgegebenheiten im Rahmen ihrer Selbstreproduktion berücksichtigen und auf diese Weise ihre Eingebundenheit in einen übergreifenden Zusammenhang thematisieren. ${ }^{30}$

Erkennt man mit Luhmann die eigenständige, selbstreferentielle Struktur der Gesellschaft wie ihrer Teilbereiche an, erscheint es damit ebensowenig sinnvoll, die auf der Grundlage reflexiver Mechanismen vermittelte Fähigkeit von Sinnsystemen, durch Selbstkonditionierung über ihre Grenzen hinweg in ihrer Umwelt Wirkungen zu erzielen, allein Individuen, Gruppen oder Organisationen zuzuschreiben. Auch die Gesellschaft und ihre Teilsysteme generieren Formen rationaler Selbstreproduktion, die sich als originäre Eigenleistung dieser Systeme nicht durch einen Rückbezug auf andere soziale Einheiten erklären.

Die Wirtschaftspolitik des Staates etwa oder die Beteiligung von Wissenschaftlern und Fachverbänden an Gesetzgebungsverfahren können in ihrer allgemeinen Erscheinungsform nur als rationale Momente der Selbstreproduktion des politischen Systems aufgefaßt werden, die sich nicht auf die Interessen einzelner politischer Gruppen, Parteien oder Regierungen reduzieren lassen und diesen nicht beliebig zur Disposition stehen. Gleiches gilt für die Entstehung ökologischer Kommunikationen bzw. entsprechender sozialer Bewegungen im Hinblick auf die Selbstreproduktion des sozialen Gesamtzusammenhangs.

Obgleich es demnach der Gesellschaft wie ihren ausdifferenzierten Teilsystemen nicht mehr gelingt, systemintern ihre Einheit konkurrenzfrei zu repräsentieren, generieren sie gleichwohl Diskurse, die die Differenz von System und Umwelt als Informationswert benutzen und sich an der Einheit dieser Unterscheidung orientieren. Trotz der fehlenden Repräsentation einer adressablen Identität läßt sich diesen Systemen damit dennoch eine spezifische, auf Grenzüberschreitungen orientier-

30 Ein selbstreferentielles System operiert in diesem Sinne nach Luhmann rational, sofern es seine Selektivität bzw. die hiermit verbundenen Einwirkungen auf seine Umwelt entlang der möglichen Rückwirkungen auf es selbst dirigiert. Die Differenz von System und Umwelt wird in diesem Fall unter dem Gesichtspunkt der Einheit dieser Differenz im System als Anschlußmöglichkeit genutzt (vgl. Luhmann 1984: 617, 638-646; 1986: 247; 1987: 114; Willke 1987a: 262 ff). Siehe auch bereits Abschnitt 3.2. te, operationale Rationalität in dem Maße zuschreiben, wie es ihnen gelingt, ihre Selbstreproduktion in der Weise auszubilden, daß sie als emergenter Zusammenhang in ihrer Umwelt Resonanzen erzeugen, um deren Rückwirkungen zur Selbstreproduktion zu nutzen.

Die Unterscheidung von handlungsfähigen und handlungsprägenden Sozialsystemen bleibt daher insoweit unzureichend, als ergänzend hierzu die besondere Qualität der reflexiven Selbstreproduktion von Sinn im Hinblick auf alle mit diesem Differenzschema selbstreferentiell operierenden Systeme Berücksichtigung finden muß. Reflexive Beobachtungen und hiermit einhergehende Versuche der Erzeugung externer Effekte stellen keine Momente dar, die ein bestimmter, handlungsfähiger Sozialtypus in einen anderen, durch Nichthandlungsfähigkeit sich auszeichnenden sozialen Sinnzusammenhang einspeist. Diesbezüglich läßt sich keine Differenz zwischen sozialen Systemen wie Wirtschaft, Politik oder Gesellschaft einerseits und Individuen, Gruppen oder Organisationen andererseits festmachen.

Vielmehr kommt an diesem Punkt unabhängig von den klassischen Bestimmungsmomenten der Handlungsfähigkeit eine analytische Unterscheidung zur Geltung, die auf alle selbstreferentiell geschlossenen Sinnstrukturen Anwendung finden kann. Dadurch wird es möglich dem Gesichtspunkt Rechnung zu tragen, daß sich ein Sinnsystem an seiner Bindung an andere Sinnstrukturen orientieren muß, während es sich zugleich in Differenz zu diesen reproduziert. Der die Systemgrenze überschreitende Aspekt bezeichnet die Eingebundenheit des Systems in einen übergreifenden Zusammenhang, die dessen fortwährende Bemühungen, über seine Grenzen hinaus auf seine Umwelt Einfluß zu nehmen und sich mit dieser zu koordinieren, hervorbringt, während eine die Systemgrenze bezeichnende Perspektive die operative Eigenselektivität des Codes, mit dem ein System sich in seiner spezifischen Individualität von seiner Umwelt unterscheidet, in den Vordergrund stellt. In dem einen Fall handelt es sich um die Rekonstruktion der Bindung eines Systems an seine Umwelt, im anderen um die Rekonstruktion der Differenz eines Systems gegenüber seiner Umwelt. Nur beide Momente zusammen ergeben ein vollständiges und befriedigendes Bild der Reproduktion psychischer und sozialer Systeme, da hierdurch ergänzend zu ihrem operativ geschlossenen Charakter sichtbar werden kann, aus welchen Gründen sie parallel zwingend intersystemische Koordinationsbemühungen hervorbringen, die 
über sie hinausweisende eigenselektive Sinnstrukturen generieren, für die sie zugleich in Form struktureller Kopplungen in besonderem Maße resonanzfähig bleiben. ${ }^{31}$

\subsection{Intersystemische Koordination und gesellschaftliche Rationalität}

Wird der Gesellschaft wie ihren Teilsystemen in dieser Weise eine emergente Form reflexiver Selbstreproduktion zugestanden, gerät ein Moment in den Blick, das insbesondere kritischen Beobachtern der Moderne zumindest Ansatzpunkte für korrektive Wirkungsmechanismen gegenüber einseitig instrumentellen Rationalisierungsprozessen bietet und ebenso Luhmanns Skepsis hinsichtlich des Rationalitätspotentials moderner Gesellschaften relativiert. ${ }^{32}$

Das Paradigma systemischer Selbstreproduktion kann, wie bereits mehrfach deutlich wurde, keineswegs bedeuten, daß soziale Systeme sich ausschließlich mit sich selbst beschäftigen. Im politischen System etwa würde eine Regierung, die gesellschaftliche Probleme wie Arbeitslosigkeit oder Umweltverschmutzung aufgrund ihrer Komplexität bzw. mangels Kontrollmöglichkeiten ausschließlich sich selbst überließe, den nächsten Wahltermin nicht überstehen. Vielmehr stellt es sich im Gegenteil so dar, daß die Politik zur Sicherung ihrer Selbstreproduktion gleichsam zwangsläufig auch ihre Umwelt zum Gegenstand bindender Entscheidung machen muß.

31 Die irreduzible Dualität der Perspektiven begründet sich aus der paradoxen Struktur der Konstitution reflexiver Identitäten, wie Habermas im Hinblick auf die Entwicklung der Ich-Identität hervorhebt: „In der Identität des Ich drückt sich das paradoxe Verhältnis aus, daß das Ich als Person überhaupt mit allen anderen Personen gleich, aber als Individuum von allen anderen schlechthin verschieden ist; in der Sprache Hegels: das Ich ist absolut Allgemeines und ebenso unmittelbar absolute Vereinzelung" (Habermas 1976: 95). Die besondere Bedeutung des über die individuelle Identität hinausweisenden sozialen Zusammenhangs begründet er ergänzend: „Das Sich-Unterscheiden von anderen muß von diesen anderen anerkannt sein“ (Habermas 1976: 93). Vgl. entsprechend auch Meads Gedanke der sozialen Konstitution individueller Identität (exemplarisch Mead 1980: $177 \mathrm{ff}$ ). Preuß (1989: 546f) hat diesen Gedanken im Hinblick auf die Operationslogik des Rechtssystems gegenüber Teubners autopoietischem Ansatz hervorgehoben.

32 Vgl. hierzu auch Bendel (1993: $129 \mathrm{ff}$ ).
Dieses Komplementärverhältnis von operativer Geschlossenheit und struktureller Umweltkopplung, das für alle Teilbereiche der Gesellschaft gleichermaßen als Bedingung ihrer Selbstreproduktion vorausgesetzt werden kann, erscheint im Rahmen der funktionalen Differenzierung der Gesellschaft als ein wechselseitiger Steigerungszusammenhang. ${ }^{33}$

D.h. parallel zur Ausbildung funktionaler Autonomie verstärkt sich gleichsam der Druck auf die Teilbereiche, rational zu operieren, um auf diese Weise eine erhöhte Resonanzfähigkeit gegenüber Umweltereignissen zu entwickeln.

Die Bedingungen etwa für Zahlen oder Nichtzahlen werden in zunehmendem Maße von globalen gesellschaftlichen Konstellationen abhängig und erfordern zur Entscheidungsfindung deren Berücksichtigung. Ein größeres Unternehmen kann es sich unter heutigen Bedingungen nicht mehr leisten, ohne die Beobachtung von Politik, Recht. Wissenschaft etc. auf dem Markt zu operieren. Es muß offen bleiben für gesellschaftliche Trends. kulturelle Entwicklungen und neue Moden, die es aufgrund der Komplexität des gesellschaftlichen Umfeldes nicht kontrollieren kann. Rechtliche Rahmenbedingungen und wissenschaftliche $\mathrm{Er}$ kenntnisse etwa erscheinen dabei im Zuge der wachsenden Differenzierung der gesellschaftlichen Teilbereiche immer weniger als eine rein externe Voraussetzung wirtschaftlichen Handelns. Sie entwickeln sich vielmehr zunehmend zu einem wesentlichen Moment des ökonomischen Investitionskalküls.

Ein besonders deutliches Beispiel bietet der Stellenwert ökologischer Fragen. Probleme des Umwelt- und Naturschutzes stellten noch vor zwanzig Jahren einen weitgehend zu vernachlässigenden Faktor bei Investitionsentscheidungen dar, während sich heute kein Unternehmen bei größeren Projekten der ökologischen Kommunikation entziehen kann.

Was die Funktionsbereiche einerseits aufgrund der Ausbildung spezifischer Kommunikationscodes an Autonomiespielräumen gewinnen, erhöht daher zugleich ihre Abhängigkeit von externen Kontexten.

33 Ein Gedanke, den bereits Durkheim und Parsons in Form des Zusammenhangs von individueller Autonomie und sozialer Bindung bzw. Universalisierung und Spezifikation im Blick hatten (vgl. exemplarisch Durkheim 1988: 183; Parsons 1967: 192-219; in Anknüpfung an Parsons auch Münch 1991). 
Mit wachsender Komplexität der Gesellschaft steigert sich somit aufgrund der enger werdenden operativen Verknüpfungen auch aus der Sicht der sich ausdifferenzierenden Teilsysteme der intersystemische Koordinationsbedarf. Um ihre Selbstreferenz zu stabilisieren, binden sie ihre Selektivität verstärkt an die Beobachtung fremdreferentieller Ereignisse in ihrer Umwelt. Sie sensibilisieren dadurch ihre Resonanzfähigkeit und konzipieren zugleich ihre Kommunikation zunehmend auch im Hinblick auf diese Abhängigkeit.

Berücksichtigt man nunmehr, daß ein System nicht allein die Beobachtung von Umweltereignissen zu seiner Selbstreproduktion nutzt, sondern aus dem gleichen Grund auch danach trachtet, aktiv auf seine Umwelt Einfluß auszuüben und Kompatibilitäten herzustellen, indem in anderen Systemen gezielt Resonanzen erzeugt werden, so generiert aus dieser die Systemgrenze überschreitenden Perspektive die funktionale Differenzierung der Gesellschaft begleitend zur operativen Geschlossenheit der Teilsysteme intersystemische Diskurse als emergente Form der Kommunikation.

Auf die gleiche Weise wie die Faszination der Kommunikation für das sie beobachtende Bewußtsein sich daraus erklärt, daß dieses zur Stabilisierung seiner Gedanken eine Bestätigung durch die Kommunikation sucht und über diesen Versuch der Abdunkelung von Kontingenzen die Kommunikation trotz deren uneinholbarer Eigenselektivität in Bewegung hält, bestimmt sich auch das Verhältnis der funktional ausdifferenzierten Teilsysteme zur Gesellschaft als übergreifende soziale Ordnung. Der umfassendere Zusammenhang erbringt eine Leistung, ohne die die Teilsysteme keine selbstreproduktive Stabilität gewinnen könnten, so daß in ihnen ein Motiv wirksam ist, die strukturelle Kopplung an die Gesellschaft trotz der parallel wirksamen Dynamik zur Steigerung ihrer operativen $\mathrm{Au}$ tonomie aufrechtzuerhalten und gezielt zu entfalten. ${ }^{34}$

34 Aus der Perspektive der Teilsysteme ist diese Abhängigkeit das ausschlaggebende Moment für die Emergenz eines eigenselektiven übergreifenden Kommunikationszusammenhangs. Diese konstitutive Bindung erklärt zugleich, warum sich die strukturellen Kopplungen zwischen sozialen Systemen nicht allein auf Formen wechselseitiger Beobachtung und strategischer Instrumentalisierung, ohne eine zusätzliche Ebene der Systembildung zu generieren, bzw. auf Formen einer teilsysteminternen Emergenz von Systemen dritter Ordnung beschränken (vgl. zur Darstellung dieser Varianten Luhmann 1990a; Teubner 1991; 1992; Krohn/Küppers 1990).
Analog dem Verhältnis der Individuen zur Kommunikation auf der Grundlage der Struktur doppelter Kontingenz erscheint die Beziehung zwischen den Funktionsbereichen dabei nicht als eine Art des unmittelbaren Austauschs zwischen Sender und Empfänger, sondern als ein eigenständiger selbstselektiver Reproduktionszusammenhang, der sich auf die universalistische Struktur sprachlicher Verständigung gründet. ${ }^{35}$

So wie zwischen die Individuen die Kommunikation als eine emergente Ebene jenseits der Selbstreferenz der beteiligten psychischen Systeme tritt, so bildet die Kommunikation zwischen den gesellschaftlichen Teilsystemen eine emergente Ebene der Gesellschaft als eine zusätzliche operativ geschlossene Sozialordnung und ebenso wie ein Bewußtseinssystem die Gedanken eines anderen nicht direkt, sondern nur im Zuge der Teilnahme am Kommunikationsgeschehen beeinflussen kann, so können sich die Teilsysteme nur mittelbar über die Teilnahme an der Kommunikation der Gesellschaft aufeinander beziehen. ${ }^{36}$

35 Die Sprache stellt soziale Ereignisse unter eigene Bedingungen der Anschlußfähigkeit. Wirtschaftliche, politische oder wissenschaftliche Kommunikationen können hier sinnvoll aneinander angeschlossen werden, da sie keiner funktionsspezifischen Selektivität unterliegen. Die Kopplung von Selektivität und Motivation vollzieht sich in diesem Fall über die in sprachlichen Äußerungen implizit erhobenen Geltungsansprüche, wobei das Erzielen von Verständigung dabei an die Stelle funktionsspezifischer Konditionierungsmuster, wie Profitorientierung, Machtgewinn oder Wahrheitssuche, tritt.

36 Das Ganze besteht nicht einfach aus der Summe seiner Teile, sondern besitzt, indem es sich als eine eigenständige selbstsubstitutive Ordnung reproduziert, zugleich eine spezifische Identität in Abgrenzung zur Operationslogik der Teilsysteme. Auch im Begriff der Gesellschaft dokumentiert sich insofern die Notwendigkeit einer irreduziblen Dualität der Perspektiven. Während er einerseits als eine Kategorie erscheint, die sich durch ihre Bestandteile charakterisiert und in dieser Weise Formen wechselseitiger Konstitution und Beeinflussung einschlieBt, bezeichnet er andererseits zugleich die Spezifik des sozialen Gesamtzusammenhangs in Differenz zu seinen Teilsystemen. Obgleich er die Gesamtheit des sozialen Lebens kennzeichnet (alle Kommunikation ist immer Kommunikation der Gesellschaft und findet nie außerhalb von ihr statt), fungiert er parallel in seiner sprachlich strukturierten Eigenart als ein Gegenpol zu den medial codierten Koordinationsformen der Teilsysteme. Der die Systemgrenze überschreitende und der die Systemgrenze bezeichnende Aspekt sind im Begriff der Gesellschaft zusammengeschlossen. 
Politische Entscheidungen oder ökonomische Investitionsvorhaben setzen sich in diesem Rahmen nicht allein durch den Verweis auf Machtkalküle oder Profitaussichten durch, sondern nur, sofern sie im Rahmen der Sinnhorizonte, die das Medium der Sprache eröffnet, Anschlußfähigkeit gewinnen.

Intersystemische Diskurse lassen sich daher grundsätzlich nur als eine dreipolige Konstellation zwischen den Teilsystemen und der Gesellschaft als übergreifendem Kommunikationssystem konzipieren. ${ }^{37}$

Die ausdifferenzierten Funktionsbereiche binden sich unter diesen Voraussetzungen aus ihrer Selbstreproduktion inhärenten Motiven an Kommunikationsbeziehungen, die über ihren selektiven Sinnhorizont hinausweisen..$^{38}$

Parallel zur Herausbildung von Momenten der Selbstorientierung treibt ihre Eigenselektivität eine die Grenzen ihrer Codes überschreitende

37 Diesem Aspekt wurde im Rahmen der Diskussion um die Möglichkeiten einer indirekten, reflexiven Kontextsteuerung bisher nicht hinreichend Rechnung getragen.

38 Ein weiterer, unmittelbar sich anschließender Punkt, den ich hier nur benennen kann, wäre die Frage, inwieweit gesellschaftliche Funktionssysteme dies in unterschiedlichem Ausmaß tun, so daß ihre Resonanzfähigkeit im Hinblick auf gesamtgesellschaftliche Problemlagen differiert, da sie ihre Selbstreproduktion nicht in gleichem Umfang auf gesamtgesellschaftliche Kommunikationshorizonte stützen. Daß dieser Aspekt sich im Rahmen der Luhmannschen Theoriekonstruktion nicht mehr sinnvoll thematisieren läßt, zeigen meines Erachtens die Überlegungen von Willke besonders deutlich. Obwohl dieser sich an die Ausgangsannahmen von Luhmann zur Struktur moderner Gesellschaften unmittelbar anlehnt, hebt er immer wieder die besonderen gesamtgesellschaftlichen Koordinationsleistungen von Politik und Recht hervor, ohne hierfür noch plausible Gründe nennen zu können. Vgl. jüngst Willke (1992: $335 \mathrm{ff}$ ), kritisch dazu jetzt auch Habermas (1992: $415 \mathrm{ff}$ ). Eine prominente Koordinationsfunktion kann einem Teilsystem nur eingeräumt werden, wenn ihm im Unterschied zu anderen Teilsystemen eine besondere Anbindung an die sprachlich strukturierten Kommunikationsprozesse der Gesellschaft attestiert wird, die ihm eine Art Transformationsfunktion zuweist, sofern erst im Zuge seiner Operationen gesamtgesellschaftliche Problemlagen in einer Weise aufgegriffen und bearbeitet werden, daß sie für andere nicht entsprechend eng an sprachliche Kommunikationen gekoppelte Teilsysteme Resonanzfähigkeit gewinnen (vgl. im Hinblick auf das Recht in diesem Sinne Habermas 1992: $77 \mathrm{f}, 108$, 429; ergänzend Peters 1993: 322 ff).
Form der Kommunikation des Systems der Gesellschaft an, die sie zwar in der Lage sind zu beeinflussen, dessen selbstselektive Struktur sprachlicher Verständigung sie jedoch nicht kontrollieren können.

Die strukturell bedingte Eigenselektivität dieses übergreifenden Kommunikationszusammenhangs, die durch die verstärkte Ausbildung rationaler Operationsmodi forciert und belebt wird. impliziert vielmehr ein autonomes Operationspotential der Gesellschaft, mit dem diese auf die Entwicklungsrichtung der Funktionsbereiche zurückwirkt und ihren Reproduktionsimperativen im Rahmen intersystemischer Diskurse Geltung verschafft.

Solche Kommunikationen produzieren daher zwangsläufig emergente Effekte, die sich nicht auf die 'Absichten' und 'Interessen' der involvierten Teilsysteme zurückführen lassen, sondern diesen gegenüber vielmehr als ein äußerer Zwang, im Sinne einer nicht beeinflußbaren Rahmenbedingung ihrer Selbstreproduktion, erscheinen, dem sie, ob es ihnen paßt oder nicht, Rechnung tragen müssen.

Obgleich ihre Beobachtung von Umweltereignissen primär von rein systemrationalen Gesichtspunkten etwa des Machtgewinns oder der Vermehrung von Zahlungsmöglichkeiten geprägt ist und Probleme gesellschaftlicher Überlebensfähigkeit keineswegs zwangsläufig mitberücksichtigt werden, markiert die Steigerung intersystemischer Abhängigkeiten in diesem Kontext ein Moment, das nicht nur funktionsspezifische Rationalitäten hervorbringt, sondern parallel rationale Selbstbeobachtungen der Gesellschaft generiert und zugleich die Resonanzfähigkeit der Teilsysteme für derartige Aspekte der Kommunikation steigert.

Investitions-, Gesetzes- oder Forschungsvorhaben können nicht mehr kommuniziert werden, ohne daß zwangsläufig eine Kommunikation unter dem Gesichtspunkt der Überlebensfähigkeit der Gesellschaft mitproduziert wird, deren Aspekte ins Kalkül der Akteure im Rahmen ihrer funktionsspezifischen Interessen einfließen müssen.

Natürlich besteht aus der Perspektive der Teilsysteme auch die Möglichkeit, unliebsame Diskussionen zu desavouieren. Statt sich etwa auf ökologische Probleme einzustellen oder Gesundheitsgefährdungen Rechnung zu tragen, können Unternehmen angesichts derartiger Problemlagen ihre Energie auch darauf verwenden, Gegenexpertisen erstellen zu lassen, um den Beweis zu erbringen. daß ein bestimmter Stoff, etwa Asbest oder 
FCKW, als nicht krebserzeugend eingestuft werden kann bzw. nicht zur Vergrößerung des Ozonlochs beiträgt.

Entscheidend ist jedoch, daß derartige Diskurse der rein systemrationalen Perspektive wirtschaftlichen Handelns entzogen sind, so daß ihre Resultate sich nicht allein unter Gesichtspunkten der Gewinnmaximierung bestimmen. Aufgrund des emergenten Charakters intersystemischer Kommunikation lassen sich die aus wirtschaftlicher Sicht wünschenswerten Effekte nicht mit der notwendigen Zuverlässigkeit hervorbringen. Desavouierungsversuche implizieren daher die Möglichkeit negativer Rückkopplungseffekte, die dann ihrerseits wirtschaftliche Ziele und Interessen diskreditieren.

Unternehmen können es sich daher nicht einfach leisten, Umweltschützer lediglich zu bekämpfen, was aus ihrer Perspektive zunächst sicherlich als die naheliegendste und billigste Lösung erscheinen mag. Vielmehr müssen sie zumindest mittelfristig ins Kalkül ziehen, daß ökologische Probleme sich zu einem marktrelevanten Faktor entwickeln, dem sie sich nicht mehr entziehen können.

Ist Asbest einmal als hochgradig krebserzeugend eingestuft oder eindeutig nachgewiesen, daß FCKW das Ozonloch vergrößern, wird es schwierig, Produkte, die diese Stoffe enthalten, zu verkaufen. Und es scheint für Unternehmen dieser Branchen ratsam, die Entwicklung dieser Diskussion nicht einfach abzuwarten bzw. sich darauf zu beschränken, eine mögliche Option zu verfolgen, wenn von vorneherein $\mathrm{zu}$ beobachten ist, $\mathrm{da}$ sich auch eine andere durchsetzen könnte.

Die prinzipielle Eigenselektivität des übergreifenden gesamtgesellschaftlichen Kommunikationszusammenhangs und die hiermit einhergehenden eigensinnigen Rationalitätskriterien führen sogar dazu, daß sich die Situation aus der Perspektive der wirtschaftlichen Akteure nicht nur als eine Art Patt zwischen verschiedenen Optionen darstellt, so daß es ratsam erscheint, sich möglichst viele offen zu halten.

Da gegenüber der gesamtgesellschaftlichen Perspektive die teilsystemspezifische immer als die eingeschränktere und singuläre erscheint, steht ein Akteur der Wirtschaft immer in der Situation, sich an übergreifende gesellschaftliche Entwicklungen und Sinnhorizonte anpassen zu müssen, während er hingegen den Verlauf dieser Kommunikation allenfalls partiell beeinflussen kann. Es empfiehlt sich demzufolge für einen systemrationalen Akteur der Wirtschaft, möglichst nah entlang des ge- samtgesellschaftlichen Rationalitätspotentials zu operieren, anstatt darauf zu hoffen, dieses einseitig in seinem Sinne beeinflussen oder gar instrumentalisieren zu können.

Aufgrund dieser Konstellation, die mit der Stellung des Bewußtseins zur Kommunikation bzw. dem Verhältnis von psychisch und sozial konstruiertem Sinn vergleichbar ist, erscheint es durchaus möglich und tendenziell sogar eher wahrscheinlich, daß sich mit der bornierten Rationalität teilsystemspezifischer Selbstreproduktion auch Momente gesamtgesellschaftlicher Rationalität verbinden. ${ }^{39}$

Obgleich die Gesellschaft ihrerseits die Operationen ihrer Teilsysteme nicht kontrollieren kann, so daß die Bedingungen für ihre Überlebensfähigkeit durch derartige operative Bindungen keineswegs zwangsläufig gesichert sind, läßt der wechselseitige Steigerungszusammenhang von operativer Autonomie und struktureller Kopplung, den Luhmann selbst immer wieder hervorhebt (vgl. Luhmann 1987: 149), dessen Annahme, die funktionale Differenzierung habe ein gesamtgesellschaftliches Rationalitätsdefizit zur Folge, zumindest in dieser grundsätzlichen Form nicht plausibel erscheinen. ${ }^{40}$

Die Auflösung der gesellschaftsinternen Einheitsrepräsentation hierarchisch organisierter Gesellschaften zugunsten einer dezentralen Struktur funktionaler Differenzierung stellt im Gegenteil,

39 Auf keinen Fall kann dies aus irgendwelchen systematischen Gründen ausgeschlossen werden, wie Kneer (1992: 104) zu Recht gegenüber Schulze-Böing/Unverferth (1986: 40) betont. Derartige Kopplungen können sicherlich nicht als eine Art unmittelbare Identität interpretiert werden, sondern eher im Sinne einer permanenten Anpassungsbewegung an eine zwangsläufig different sich reproduzierende Sinnstruktur.

40 Dieser Gedanke erinnert vielmehr an von ihm kritisierte Vorstellungen gesellschaftlicher Differenzierung als eine Art Nullsummenspiel. Luhmann ist allerdings, wie man weiß, mit solchen Diagnosen sehr vorsichtig und zurückhaltend. Er spricht daher nicht dezidiert von gesamtgesellschaftlichen Rationalitätsdefiziten. Gleichwohl meine ich, daß seine Beschreibungen der modernen Gesellschaft, soweit sie das Verhältnis der funktional ausdifferenzierten Teilsysteme zum sozialen Gesamtzusammenhang betreffen, in diese Richtung zu interpretieren sind, ohne damit gleichzeitig bereits festzulegen, inwieweit es sich dabei um beklagenswerte oder kritikwürdige Zustände handelt. Vgl. entsprechende Textpassagen bei Luhmann (1984: 621, 645; 1986: 257; 1988: 340 f; 1990: 696). 
so ist anzunehmen, eher einen Gewinn an gesellschaftlichen Reflexionsmöglichkeiten dar, da in diesem Fall nicht mehr ein System sich stellvertretend für alle anderen gesellschaftlich relevanten Problemlagen zuwendet, so daß diese nur auf dem (Um-)Weg über diesen Teilbereich bearbeitet werden können, während andere Bereiche von dieser 'Sorge' entlastet bleiben.

Jedes ausdifferenzierte Funktionssystem steht unter diesen Bedingungen vielmehr vor der Aufgabe, aus seiner Sicht die Gesellschaft und deren Umwelt zu beobachten und im Rahmen seiner Selbstreproduktion diesen Beobachtungen Rechnung zu tragen. Das 'Problem der Gesellschaft' entzündet sich im Zuge gesteigerter Differenzierungsprozesse gleichsam zunehmend an den Verweisungshorizonten jeder Kommunikation und kann nicht mehr auf besondere Teilbereiche beschränkt werden. ${ }^{41}$

Die Wirtschaft beispielsweise ist aus diesem Grund nicht in der Lage, ihre Verantwortung für ökologische Probleme einfach auf andere Bereiche wie etwa die Politik und deren gesellschaftliche Steuerungskompetenzen abzuschieben. Unternehmen ist es bei Investitionsvorhaben nicht einfach möglich zu behaupten, der Umweltschutz sei ihre Sache nicht, da sie sich vornehmlich mit der Förderung des materiellen Reichtums und der Schaffung von Arbeitsplätzen beschäftigten, gleichgültig, ob die Gesellschaft dabei in ihrer Überlebensfähigkeit gefährdet wird oder nicht.

Zur Entfaltung eines gesellschaftlichen Rationalitätspotentials bedarf es somit keineswegs unbedingt einer konkurrenzfreien internen Repräsentation der Einheit der Gesellschaft durch eines ihrer Subsysteme, sondern lediglich eines Typus der Kommunikation, der sich an der Einheit der Differenz von Gesellschaft und Umwelt orientiert und dabei strukturell an die Selbstreproduktion der Teilsysteme gekoppelt bleibt.

Entscheidend hierfür ist insofern ausschließlich der Erhalt der Gesellschaft als ein emergentes, selbstreproduktives Sozialsystem, das sich ebenso wie alle anderen sozialen Systeme im Zuge ver-

41 Der feste klar lokalisierte Ort gesellschaftsrationaler Kommunikation verflüchtigt sich ebenso wie ein konkret sich festschreibenlassender Inhalt, so daß sich, in Anlehnung an eine Formulierung von Habermas, die vernünftige Identität komplexer Gesellschaften lediglich „an der Struktur dieses Erzeugungsprozesses, d.h. an den formalen Bedingungen des Zustandekommens und der Überprüfung" (Habermas 1976: 107) bemißt. stärkter Differenzierungsprozesse zu rationalen Operationsmodi gedrängt sieht.

Eine Gefährdung dieser Ebene gesamtgesellschaftlicher Kommunikation durch die Verselbständigungsdynamik der Teilbereiche scheint jedoch unter diesen Voraussetzungen grundsätzlich insoweit ausgeschlossen, als es trivialerweise kein selbstreferentielles Funktionssystem ohne übergreifenden, eigenselektiven gesellschaftlichen Kommunikationszusammenhang geben kann. Ebenso wie Bewußtsein ohne Kommunikation sind Geld und Macht ohne Sprache undenkbar. ${ }^{42}$

Es erscheint insofern grundsätzlich verfehlt, intersystemische Diskurse ausschließlich auf der Grundlage rein strategischer Nutzenkalküle bzw. zweckrationaler Partikularperspektiven im Rahmen der medial codierten Reproduktion der gesellschaftlichen Teilsysteme zu rekonstruieren. ${ }^{43}$

42 Wie bereits angedeutet soll die Möglichkeit gesellschaftlicher Rationalitätsdefizite, etwa im Hinblick auf ökologische Fragen, damit keineswegs ausgeschlossen werden. Ihr Nachweis bleibt eine empirisch zu verifizierende Frage. Vielmehr möchte ich lediglich darauf hinweisen, daß sich der Ort ihrer Thematisierung durch Prozesse funktionaler Differenzierung und Spezifikation nicht verflüchtigt.

43 Dies ist der meines Erachtens zutreffende, zentrale Einwand, den Habermas gegen die Vorstellung intersystemischer Diskurse auf der Grundlage einer vollständig geschlossenen Selbstreproduktion der beteiligten Systeme formuliert (vgl. Habermas 1992: $415 \mathrm{ff}$ ). Die mit der These operativer Geschlossenheit und wechselseitiger Intransparenz vollzogene Reduktion intersystemischer Kommunikationsbeziehungen auf die Form wechselseitiger Beobachtungen steht vor der Schwierigkeit, die Koordinationsfunktion intersystemischer Diskurse in ein gesellschaftstheoretisches Gesamtkonzept zu integrieren, da dem Charakter der Sprache als ein spezifische Codes konstitutiv fundierendes Kommunikationsmedium nicht mehr Rechnung getragen werden kann. Entsprechend steht auch Teubners Versuch, gesellschaftsinterne strukturelle Kopplungen (Interferenz) als ein über wechselseitige Beobachtungsbeziehungen hinausgehendes „Prozessieren von Sinnmaterialien über Systemgrenzen hinweg" (Teubner 1989: 110) zu interpretieren vor der Frage, inwieweit dieses Konzept noch mit dem Kern des Autopoiesistheorems kompatibel ist (vgl. exemplarisch Teubner 1989: $102 \mathrm{ff}$; kritisch hierzu Habermas 1992: $73 \mathrm{ff}$ sowie Preuß 1989: 540ff; ausführlich zu dieser Frage Bendel 1993: $114 \mathrm{ff}$ ). Behält man hingegen die vorgängige und grundlegende Funktion der Sprache im Auge, erscheint es jedoch gleichwohl instruktiv, die Formen intersystemischer Koordination aus der Perspektive der Teilsysteme zu rekonstruieren. Sicherlich ist es zutreffend, daß diese aufgrund ihrer spezifischen 
Symbolisch generalisierte Kommunikationscodes operieren trotz ihrer eigensinnigen Rationalitätshorizonte, die eine Struktur wechselseitiger Intransparenz und Verständnislosigkeit erzeugen, auf der Basis einer gemeinsamen Anbindung an eine die selektiven Perspektiven der Teilsysteme transzendierende Sprache, die aufgrund ihrer universalistischen Struktur den Raum für intersystemische Koordinationsprozesse eröffnet.

Nur sofern man ergänzend zur operativen Autonomie der Teilsysteme und den Formen einer rein zirkulären wechselseitigen Beobachtung eine Ebene struktureller Kopplung an einen emergenten, gesamtgesellschaftlichen Kommunikationszusammenhang vorsieht, die auf der Grundlage der Unterscheidung von Code und Programm ein Moment der Übertragung von Sinn über Systemgrenzen hinweg einschließt, lassen sich Fragen der CoEvolution und Koordination sinnvoll thematisieren.

Mit dem Konzept der Gesellschaft als ein eigenständiger, im Medium alltagssprachlicher Verständigung sich entfaltender Kommunikationszusammenhang, an dessen emergente Eigenselektivität die auf der Basis spezialisierter Codes operierenden Teilsysteme gleichsam als Bedingung ihrer Ausdifferenzierung konstitutiv gebunden sind, läßt sich zugleich eine Lücke im Rahmen der Theorie sozialer Systeme schließen, die daraus zu resultieren schien, daß die dezentrale Struktur funktional differenzierter Gesellschaften keinem Teilsystem die privilegierte Wahrnehmung gesellschaftsweiter Koordinations- und Integrationsfunktionen einräumt.

Selektivität aus sich heraus nicht dazu in der Lage sind, Sinnhorizonte im Hinblick auf gesamtgesellschaftlich relevante Problemlagen zu mobilisieren. Anhand dieses Blickwinkels läßt sich jedoch verdeutlichen, inwieweit funktionsspezifische Kommunikationscodes ihrerseits immanent auf die Formen einer gesellschaftsweiten Kommunikation verweisen. Gesellschaftsrationale Kommunikationen müssen sich unter Berücksichtigung dieses Aspekts nicht einfach von außen im Rahmen autonomer Öffentlichkeiten und zivilgesellschaftlicher Assoziationen (vgl. hierzu Habermas 1992: 399 ff) gegen funktionsspezifisch bornierte Orientierungen behaupten. Vielmehr können sie an interne, ihnen sozusagen entgegenkommende Rationalitätshorizonte der Teilsysteme anknüpfen. Zugleich wird dadurch deutlich, daß die Entwicklungsdynamik funktionaler Differenzierung sich nicht in einem Prozeß der Austrocknung von Lebenswelten erschöpft.

\section{Literatur}

Baecker, D., 1992: Die Unterscheidung zwischen Kommunikation und Bewußtsein. S. 217-268 in: W. Krohn/ G. Küppers (Hrsg.), Emergenz: Die Entstehung von Ordnung, Organisation und Bedeutung. Frankfurt a.M.: Suhrkamp.

Bendel, K., 1993: Selbstreferenz, Koordination und gesellschaftliche Steuerung. Zur Theorie der Autopoiesis sozialer Systeme bei Niklas Luhmann. Pfaffenweiler: Centaurus.

Bender. C., 1989: Identität und Selbstreflexion. Zur reflexiven Konstruktion der sozialen Wirklichkeit in der Systemtheorie von $\mathrm{N}$. Luhmann und im symbolischen Interaktionismus von G.H. Mead. Frankfurt a.M./ Bern/New York: P. Lang.

Durkheim, E., 1988: Über soziale Arbeitsteilung. Studie über die Organisation höherer Gesellschaften. Frankfurt a.M.: Suhrkamp.

Eichmann, R., 1989: Diskurs gesellschaftlicher Teilsysteme. Zur Abstimmung von Bildungssystem und Beschäftigungssystem. Wiesbaden: Deutscher Universitäts-Verlag.

Foerster, H.v., 1985: Sicht und Einsicht. Versuche zu einer operativen Erkenntnistheorie. Braunschweig: Vieweg.

Fuchs, P., 1992: Die Erreichbarkeit der Gesellschaft. Zur Konstruktion und Imagination gesellschaftlicher Einheit. Frankfurt a.M.: Suhrkamp.

Giegel, H.-J., 1987: Interpenetration und reflexive Betimmung des Verhältnisses von psychischen und sozialen Systemen. S. 212-244 in: H. Haferkamp/M. Schmid (Hrsg.), Sinn, Kommunikation und soziale Differenzierung. Frankfurt a.M.: Suhrkamp.

Habermas, J., 1976: Können komplexe Gesellschaften eine vernünftige Identität ausbilden? S. 92-126 in: J. Habermas, Zur Rekonstruktion des Historischen Materialismus. Frankfurt a.M.: Suhrkamp.

Habermas, J., 1981: Theorie kommunikativen Handelns. 2 Bde. Frankfurt a.M.: Suhrkamp.

Habermas, J., 1992: Faktizität und Geltung. Beiträge zur Diskurstheorie des Rechts und des demokratischen Rechtsstaats. Frankfurt a.M.: Suhrkamp.

Heidenescher, M., 1992: Zurechnung als soziologische Kategorie. Zu Luhmanns Verständnis von Handlung und Systemleistung. Zeitschrift für Soziologie 21: 440-455.

Kneer, G., 1992: Bestandserhaltung und Reflexion. Zur kritischen Reformulierung gesellschaftlicher Rationalität. S. 86-112 in: M. Krawietz/M. Welker (Hrsg.), Kritik der Theorie sozialer Systeme. Frankfurt a.M.: Suhrkamp.

Krohn, W./Küppers, G., 1990: Selbstreferenz und Planung. S. 109-127 in: Selbstorganisation. Jahrbuch für Komplexität in den Natur-, Sozial- und Geisteswissenschaften. Bd. 1. Berlin: Duncker \& Humblot.

Krohn, W./Küppers, G. (Hrsg.), 1992: Emergenz: Die Entstehung von Ordnung, Organisation und Bedeutung. Frankfurt a.M.: Suhrkamp.

Luhmann, N., 1984: Soziale Systeme. Grundriß einer allgemeinen Theorie. Frankfurt a.M.: Suhrkamp.

Luhmann, N., 1985: Einige Probleme mit dem 'reflexiven Recht'. Zeitschrift für Rechtssoziologie 6: 1-18. 
Luhmann, N., 1985a: Die Autopoiesis des Bewußtseins. Soziale Welt 36: 402-446.

Luhmann, N., 1986: Ökologische Kommunikation. Kann die moderne Gesellschaft sich auf ökologische Gefährdungen einstellen? Opladen: Westdeutscher Verlag.

Luhmann, N., 1986a: Systeme verstehen Systeme. S. 72117 in: N. Luhmann/K.E. Schorr (Hrsg.), Zwischen Intransparenz und Verstehen. Fragen an die Pädagogik. Frankfurt a.M.: Suhrkamp.

Luhmann, N., 1987: Machtkreislauf in Recht und Demokratien. S. 142-151 in: Soziologische Aufklärung. Bd. 4. Opladen: Westdeutscher Verlag.

Luhmann, N., 1987a: Sozialisation und Erziehung. S. 173-181 in: Soziologische Aufklärung. Bd. 4. Opladen: Westdeutscher Verlag.

Luhmann, N., 1987b: Codierung und Programmierung. Bildung und Selektion im Erziehungssystem. S. 182201 in: Soziologische Aufklärung. Bd. 4. Opladen: Westdeutscher Verlag.

Luhmann, N., 1987c: Closure and Openness: On Reality in the World of Law. S. 335-348 in: G. Teubner (Hrsg.), Autopoietic Law: A New Approach to Law and Society. Berlin: de Gruyter.

Luhmann, N., 1988: Die Wirtschaft der Gesellschaft. Frankfurt a.M.: Suhrkamp.

Luhmann, N., 1988a: Wie ist BewuBtsein an der Kommunikation beteiligt? S. 884-905 in: H.U. Gumbrecht/ K.L. Pfeiffer (Hrsg.), Materialität der Kommunikation. Frankfurt a.M.: Suhrkamp.

Luhmann, N., 1989: Politische Steuerung: Ein Diskussionsbeitrag. Politische Vierteljahreszeitschrift 30: 4-9.

Luhmann, N., 1990: Die Wissenschaft der Gesellschaft. Frankfurt a.M.: Suhrkamp.

Luhmann, N., 1990a: Verfassung als evolutionäre Errungenschaft. Rechtshistorisches Journal 9: 176-220.

Luhmann, N., 1991: Soziologie des Risikos. Berlin/New York: de Gruyter.

Luhmann, N., 1991a: Steuerung durch Recht? Einige klarstellende Bemerkungen. Zeitschrift für Rechtssoziologie 12: 142-146.

Luhmann, N., 1992: Wer kennt Wil Martens? Kölner Zeitschrift für Soziologie und Sozialpsychologie 44: 140142.

Martens, W., 1991: Die Autopoiesis sozialer Systeme. Kölner Zeitschrift für Soziologie und Sozialpsychologie 43: 625-646.

Martens, W., 1992: Die partielle Überschneidung autopoietischer Systeme. Eine Erwiderung. Kölner Zeitschrift für Soziologie und Sozialpsychologie 45: 143145.

Maturana, H., 1982: Erkennen. Die Organisation und Verkörperung von Wirklichkeit. Braunschweig: Vieweg.

Mayntz, R., 1987: Politische Steuerung und gesellschaftliche Steuerung - Anmerkungen zu einem theoretischen Paradigma. S. 89-110 in: T. Ellwein/J.J. Hesse/R. Mayntz/F.W. Scharpf (Hrsg.), Jahrbuch zur Staats- und Verwaltungswissenschaft. Bd. 1. Baden-Baden: Nomos.

Mead, G.H., 1980: Geist, Identität und Gesellschaft. Frankfurt a.M.: Suhrkamp.

Miller, M., 1987: Selbstreferenz und Differenzerfahrung. Einige Überlegungen zu Luhmanns Theorie sozialer Systeme. S. 187-211 in: H. Haferkamp/M. Schmid
(Hrsg.), Sinn, Kommunikation und soziale Differenzierung. Frankfurt a.M.: Suhrkamp.

Münch, R., 1991: Dialektik der Kommunikationsgesellschaft. Frankfurt a.M.: Suhrkamp.

Parsons, T., 1967: Pattern Variables Revisited. S. 192-219 in: T. Parsons, Sociological Theory and Modern Society. New York: Free Press.

Parsons, T., 1968: The Structure of Social Action. New York: Free Press.

Peters, B., 1993: Die Integration moderner Gesellschaften. Frankfurt a.M.: Suhrkamp.

Pokol, B., 1990: Professionelle Institutionensysteme oder Teilsysteme der Gesellschaft? Reformulierungsvorschläge zu Niklas Luhmanns Systemtypologie. Zeitschrift für Soziologie 19: 329-347.

PreuB, U.K., 1989: Rationality Potentials of Law. Allocative, Distributive and Communicative Rationality. S. 525-555 in: C. Joerges/D.M. Trubek (Eds.), Critical Legal Thought: An American-German Debate. BadenBaden: Nomos.

Scharpf, F.W., 1989: Politische Steuerung und politische Institutionen. Politische Vierteljahresschrift 30: 10-21.

Schimank, U., 1985: Der mangelnde Akteurbezug systemtheoretischer Erklärungen gesellschaftlicher Differenzierung - Ein Diskussionsvorschlag. Zeitschrift für Soziologie 14: 412-434.

Schimank, U., 1988: Gesellschaftliche Teilsysteme als Akteurfiktionen. Kölner Zeitschrift für Soziologie und Sozialpsychologie 40: 619-639.

Schmidt, S.J. (Hrsg.), 1987: Der Diskurs des Radikalen Konstruktivismus. Frankfurt a.M.: Suhrkamp.

Schulze-Böing, M./Unverferth, H.-J., 1986: Rationalität in komplexen Sozialsystemen. S. 14-90 in: H.-J. Unverferth (Hrsg.), System und Selbstreproduktion. Frankfurt a.M./Bern/New York: P. Lang.

Teubner, G., 1987: Hyperzyklus in Recht und Organisation. Zum Verhältnis von Selbstbeobachtung, Selbstkonstitution und Autopoiese. S. 89-128 in: H. Haferkamp/M. Schmid (Hrsg.), Sinn, Kommunikation und soziale Differenzierung. Frankfurt a.M.: Suhrkamp.

Teubner, G., 1989: Recht als autopoietisches System. Frankfurt a.M.: Suhrkamp.

Teubner, G., 1991: Steuerung durch plurales Recht. Oder: Wie die Politik den normativen Mehrwert der Geldzirkulation abschöpft. S. 528-551 in: W. Zapf (Hrsg.), Die Modernisierung moderner Gesellschaften. Verhandlungen des 25. deutschen Soziologentages in Frankfurt a.M. 1990. Frankfurt a.M./New York: Campus.

Willke, H., 1987: Strategien der Intervention in autonome Systeme. S. 333-361 in: D. Baecker/J. Markowitz/R. Stichweh/H. Tyrell/H. Willke (Hrsg.), Theorie als Passion. Frankfurt a.M.: Suhrkamp.

Willke, H., 1987a: Differenzierung und Integration in Luhmanns Theorie sozialer Systeme. S. 247-274 in: H. Haferkamp/M. Schmid (Hrsg.), Sinn, Kommunikation und soziale Differenzierung. Frankfurt a.M.: Suhrkamp.

Willke, H., 1989: Systemtheorie entwickelter Gesellschaften. Dynamik und Riskanz moderner gesellschaftlicher Selbstorganisation. Weinheim/München: Juventa.

Willke, H., 1992: Ironie des Staates. Grundlinien einer Staatstheorie polyzentrischer Gesellschaft. Frankfurt a.M.: Suhrkamp. 